\title{
Survival advantages of multicellular spheroids vs. monolayers of HepG2 cells in vitro
}

\author{
CHUN-LI LI ${ }^{1}$, TAO TIAN ${ }^{1}$, KE-JUN NAN ${ }^{1}$, NA ZHAO ${ }^{2}$, YA-HUAN GUO ${ }^{1}$, \\ $\mathrm{JIE} \mathrm{CUI}^{1}, \mathrm{JIN} \mathrm{WANG}^{1}$ and WANG-GANG ZHANG ${ }^{3}$
}

\begin{abstract}
${ }^{1}$ Department of Medical Oncology, The First Affiliated Hospital of The School of Medicine of Xi'an Jiaotong University, Xi'an 710061, Shaanxi Province, ${ }^{2}$ Department of Medicine, The Central Hospital of Nanhui District, Shanghai 210300, ${ }^{3}$ Department of Medical Hematology, The Second Affiliated Hospital of The School of Medicine of Xi'an Jiaotong University, Xi'an 710000, Shaanxi Province, P.R. China
\end{abstract}

Received July 24, 2008; Accepted August 29, 2008

DOI: $10.3892 /$ or_00000167

\begin{abstract}
Mammalian cells grow in three-dimensions (3-D) in vivo. Commonly used two-dimensional (2-D) cell cultures are inadequate to recreate the biological microenvironment of tumor cells. The potentially different outcomes from 2-D and 3-D culture systems may have a significant impact on the relevance of experimental findings. The purpose of this study was to characterize the human hepatoma cell line HepG2 in 2-D and 3-D cultures. HepG2 cells in 2-D and 3-D cultures were treated with cisplatin, 5-fluorouracil, and adriamycin and were analyzed by scanning electron microscopy and transmission electron microscopy. Cell cycle progression and apoptosis were detected by flow cytometry. Inhibition of cell proliferation was quantified by MTT assay. The expression of E-cadherin, CD44v6, VEGF, KDR, endostatin, Bax, and cytochrome-c were analyzed by immunohistochemical (IHC) staining. As compared to the 2-D monolayer culture, the 3-D multicellular spheroids (MCS) of HepG2 cells featured a greater fraction of cells in G1 phase and were organized with more abundant cell-cell adhesion. In addition, cells in MCS were significantly less apoptotic in maintenance culture media and were more resistant to drug-induced apoptosis. Ecadherin, CD44v6, VEGF, KDR, endostatin, and cytochrome-c levels were increased in MCS as compared to 2-D cell cultures. In coclusion, MCS conferred differ-entiated phenotypes including increased cell-cell adhesion and G1 phase cell cycle arrest, enhanced cellular resistance to apoptosis, and upregulated angiogenic potential. Based on our data, a multicellular morphological hierarchy may sustain
\end{abstract}

Correspondence to: Dr Chun-Li Li, Department of Medical Oncology, The First Affiliated Hospital of The School of Medicine of Xi'an Jiaotong University, Xi'an 710061, Shaanxi Province, P.R. China

E-mail: chunli5158@163.com

Key words: hepatocellular carcinoma, adhesion, apoptotic sensitivity, cell cycle progression, angiogenesis, 2-D culture, 3-D culture, multicellular spheroids the growth/survival advantages of cancer cells in vivo. Therefore, a 3-D culture system should be the preferred technique for cancer biology investigation.

\section{Introduction}

Hepatocellular carcinoma (HCC) is the most common malignancy of the liver. It is the third most common cause of cancer mortality globally, and thus a major health concern worldwide. Therapeutic options for HCC include surgical resection, local ablative therapies, systemic treatment, and liver transplantation (1). Surgery, being the most effective treatment, is unfortunately not applicable to the majority of HCC patients since the diagnosis is most often made at unresectable stages. Even for patients eligible for hepatic resection, the postoperative recurrence rate is as high as $50 \%$ two years after surgery. As a result, the median life span of HCC patients after diagnosis is $<6$ months (2). Chemotherapy is an area that has yet to be explored as an alternative to improve the prognosis of HCC. Unfortunately, current chemotherapies for HCC generally have low response rates (3-6). It is important to note that cytotoxicity in established cancer cell lines cultured in a two-dimensional (2-D) monolayer is a standard first line drug screening criterion. However, as suggested by clinical observations, HCC, in their primary and disseminated states, tend to be multicellular. More importantly, tumor cells always grow in a three-dimensional (3-D) space in vivo. Thus, the commonly used 2-D cell culture system is probably inadequate to recreate the biological microenvironment of tumor cells (7). The potentially different outcomes from 2-D and 3-D culture systems may have a significant impact on the relevance of experimental findings.

In light of this, 3-D culture has gained significant approval as a more advantageous in vitro system than $2-\mathrm{D}$ culture, for studying various molecular processes and screening therapeutic agents. In the 3-D culture model, many established human cancer cell lines form multicellular spheroids (MCS) which mimic solid tumors more closely than the monolayer cells in 2-D cultures (8). The 3-D system seems to be particularly valuable in the assessment of tumor cell drug 
sensitivity since MCS manifest elevated resistance to chemotherapeutic agents compared with monolayer cell cultures. The increased drug resistance of MCS may be a result of increased cell-cell interactions and polarized cellmatrix interactions, which may not be supported by the monolayer cell culture system. It is well documented that molecules that regulate cell-cell and cell-matrix interactions, including E-cadherin, catenins, CD44 family members and integrin, play critical roles in cell proliferation, differentiation and apoptosis (9). In addition to the histological organization and the intrinsic characteristics of tumor cells, tumor angiogenesis also regulates drug resistance. To this end, tumor cells are considered to be the major contributor of the angiogenic growth factor, vascular endothelia growth factor (VEGF). It is intriguing to speculate that MCS may provide a convenient in vitro model to screen and characterize anti-cancer drugs and to study how tumor cells may contribute to angiogenic responses in their natural environment. These possibilities are addressed in the current study.

\section{Materials and methods}

2-D and 3-D cell culture. The human hepatoma cell line HepG2 (ATCC, USA) was maintained in a 2-D monolayer culture in RPMI-1640 supplemented with $10 \%$ fetal bovine serum (Gibco, USA). MCS of HepG2 cells were obtained by a liquid overlay technique as described previously (10). Briefly, for 2-D cultures, a HepG2 single cell suspension in complete media was seeded at $2 \times 10^{5}$ cells $/ \mathrm{ml}$ in each culture flask. The conditions for 3-D cell culture were the same as for monolayer cultures, except that the culture flasks were coated with $2 \%$ agarose before cell plating. Fifty percent of the culture medium was renewed every two days.

Scanning electron microscopy (SEM) and transmission electron microscopy (TEM) studies of 2-D and 3-D cell organization. The monolayer cells and MCS were washed with PBS and fixed in $2.5 \%$ glutaraldehyde for $2 \mathrm{~h}$. The cells were then post-fixed on the plate with $1 \% \mathrm{OsO}_{4}$, and dehydrated by graded ethanol. The cells were then covered with gold palladium and examined by SEM (JEOL, JSM-840, Japan), or embedded in Epon812 expoy resin and examined by TEM (Hitachi, H-600, Japan).

MTT cell viability assay. The cytotoxicity of drugs was measured by 3-(4,5-dimethylthiazol-2-yl)-2, 5-diphenyl tetrazolium bromide (MTT) assay using a previously described method (11). Briefly, 1500 cells in either a monolayer or MCS in $150 \mu \mathrm{l}$ of maintenance medium were seeded into a 96-well plate. Next, the cells were treated with $50 \mu 1$ of cisplatin (CDDP) (Qilu Pharmaceutical Company, Shandong, P.R. China), 5-fluorouracil (5-FU) (Xudonghaipu Pharmaceutical Company, Shanghai, P.R. China), or adriamycin (ADM) (Haizheng Pharmaceutical Company, Zhejiang Province, P.R. China) for $48 \mathrm{~h}$ at the indicated concentrations. The cells were then incubated with $20 \mu \mathrm{l}$ of MTT reagent $\left(5 \mathrm{mg} / \mathrm{ml}\right.$, Sigma, USA) at $37^{\circ} \mathrm{C}$ for $4 \mathrm{~h}$. The media was collected and measured at the spectrometric absorbance of $492 \mathrm{~nm}$, yielding $\mathrm{A}_{492 \mathrm{~nm}}$ values, using a Multifunction microplate reader (POLARstarOPTIMA, BMG,
Germany). The inhibition of cell viability was calculated by the formula $\left(1-\mathrm{A}_{492 \mathrm{~nm}} / \mathrm{A}_{492 \mathrm{~nm}}^{\prime}\right) \times 100 \%$, where $\mathrm{A}_{492 \mathrm{~nm}}$ is from the treated cells and $A_{492 \mathrm{~nm}}^{\prime}$ is from the untreated cells. All sample measurements were replicated 5 times.

Cell sorting by flow cytometry. Monolayer cells and MCS were harvested, suspended, and fixed in $70 \%$ alcohol at $4^{\circ} \mathrm{C}$ overnight. The cells were then stained by propidium iodide (PI, $50 \mu \mathrm{g} / \mathrm{ml}$ ) at $37^{\circ} \mathrm{C}$ in the dark for $30 \mathrm{~min}$ and analyzed by flow cytometer (FACScan, BD, USA). In parallel, cells harvested from monolayer or MCS cultures were stained with PI and assayed for Annexin V. Briefly, cells were resuspended in a $200 \mu \mathrm{l}$ solution containing FITC-conjugated Annexin V antibody (Beijing Zhongshan Golden Bridge Company, P.R. China) and PI $(50 \mu \mathrm{g} / \mathrm{ml})$ for $15 \mathrm{~min}$ and analyzed by flow cytometry. The percentage of Annexin Vpositive/PI-negative apoptotic cell populations was calculated using the Cell Quest software.

$I H C$. HepG2 cells were plated on glass coverslips in a monolayer and fixed with $4 \%$ polyoxymethylene. The MCS were fixed with $4 \%$ polyoxymethylene and embedded in paraffin. Blocks were sectioned at a thickness of $4 \mu \mathrm{m}$, deparaffinized and rehydrated in graded alcohol. After quenching endogenous peroxidase with $3 \% \mathrm{H}_{2} \mathrm{O}_{2}$ in methanol for $30 \mathrm{~min}$, the sections were incubated overnight at $4^{\circ} \mathrm{C}$ with mouse monoclonal antibodies against human E-cadherin (1:200 dilution), CD44v6 (1:200 dilution) (Maixin Biotechnology Co.), VEGF (1:200 dilution), VEGFR-2 (1:200 dilution), endostatin (1:200 dilution), Bax (1:200 dilution), or cytochrome-c (1:200 dilution). The antibodies against E-cadherin and CD44v6 were from P.R. China. All other primary antibodies were from Santa Cruz Biotechnology Inc, (Santa Cruz, CA, USA). After the slides were blocked, the sections were incubated with the antimouse secondary antibody for $40 \mathrm{~min}$ at $37^{\circ} \mathrm{C}$. The bound secondary antibody was visualized by the activity of the horse radish peroxidase conjugate using a 3,3-diaminobenzidine tetrahydrochloride substrate (Sigma, UK). The sections were counter-stained with hematoxylin and fixed. For semi-quantitative evaluation of the IHC, 20 random visual fields were examined using the LeicaQ550cw imaging analysis system (Germany).

Statistical analysis. Data were reported as means \pm SD. The paired t-test was used for statistical analysis, and $\mathrm{p}<0.05$ was considered statistically significant.

\section{Results}

Morphological characteristics. After 3-4 days of incubation, monolayer cells were adhered to the bottom of the flasks and showed significant cell body extension, while MCS were floating in the media (Fig. 1A). SEM showed that the MCS were in different sizes, ranging from 160-200 $\mu \mathrm{m}$ in diameter and their shapes were either oval spheroid, polyhedron or tightly packed (Fig. 1B). TEM showed abundant desmosome and intermediate junctions only in MCS. In parallel, the monolayer cells were typically polygonal-shaped with more microvilli on their surfaces, and had wider intercellular spaces 


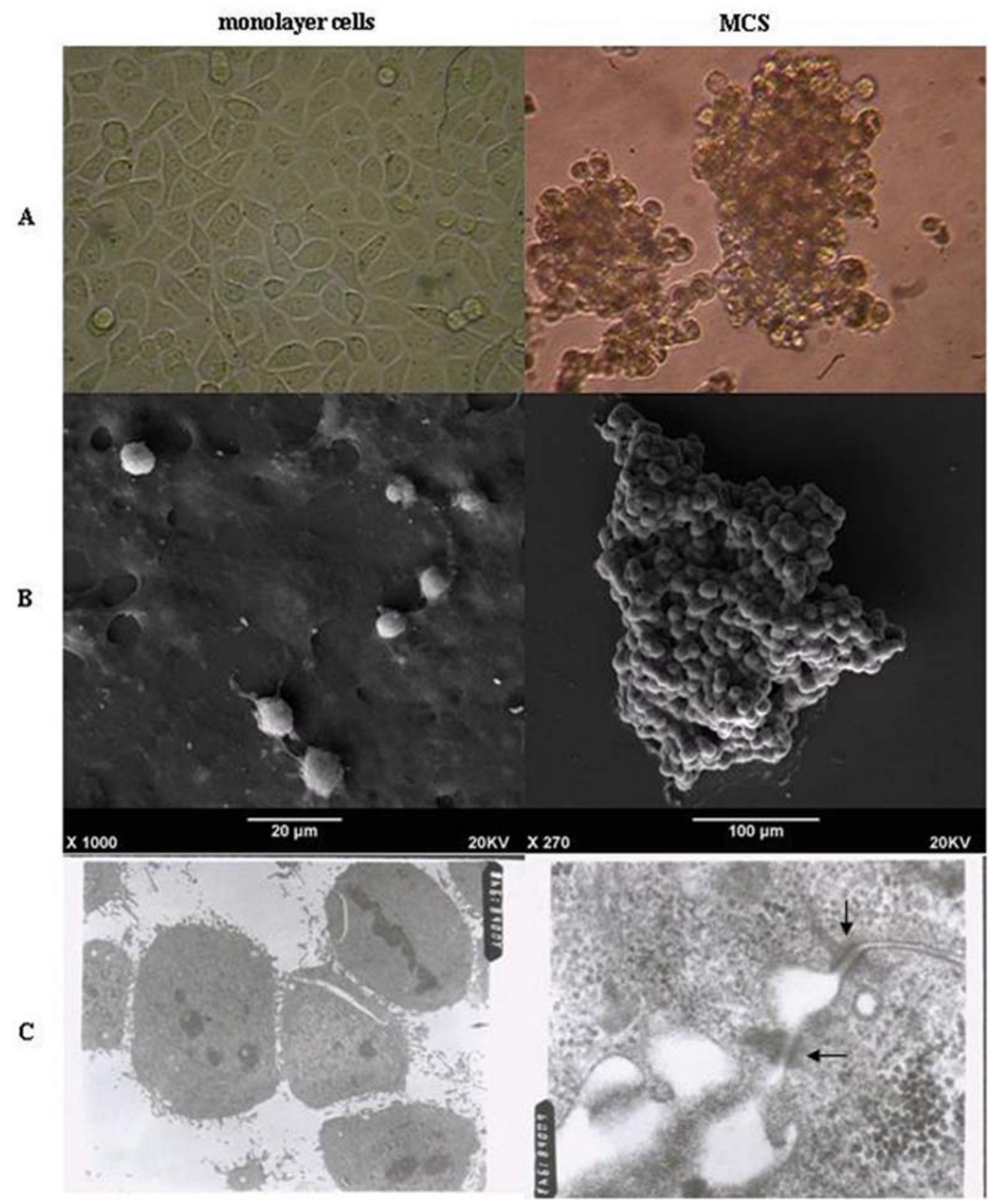

Figure 1. Morphological characterization of 2-D and 3-D HepG2 cell cultures. (A) Phase contract microscopy, x100. (B) Scanning electron microscopy, x30000 and (C) transmission electron microscopy, x60000.

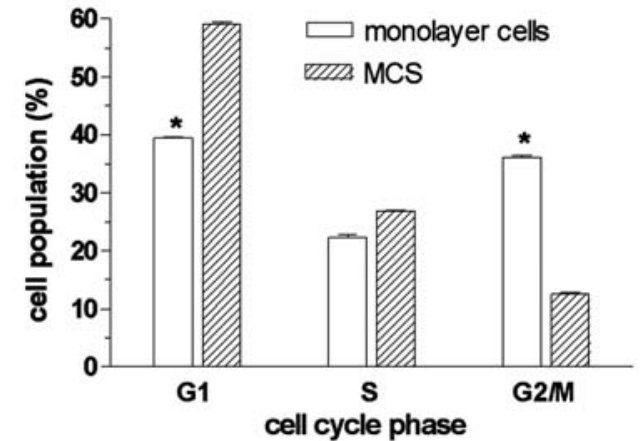

Figure 2. The effects of cellular organization on HepG2 cell cycle progression. Summarizes the quantitative differences of monolayer cultures and MCS in different phases of the cell cycle. Each experiment was repeated 5 times $\left({ }^{*} \mathrm{p}<0.05\right)$ compared to those in the MCS. Interestingly, cells in MCS had significantly higher numbers of mitochondria than monolayer cells (Fig. 1C).

The relationship of multicellular organization to proliferation and apoptosis. As shown in Fig. 2, more cells in the MCS accumulated in the G1 phase of the cell cycle $(59.23 \pm$ $0.41 \%)$ as compared to the monolayer cells $(39.45 \pm 0.27 \%)$ $(\mathrm{p}<0.01)$. Consequently, the MCS had a smaller cell population in the $\mathrm{G} 2$ phase $(12.47 \pm 0.65 \%)$ than the monolayer cells $(36.15 \pm 0.69 \%)(\mathrm{p}<0.01)$. The percentage of cells in $\mathrm{S}$ phase was similar between monolayer cultures $(22.37 \pm 0.76 \%)$ and MCS $(26.82 \pm 0.18 \%)(p>0.05 \%)$. MCS also have altered kinetics of drug-induced cell death. Specifically, the early stage cell death (ED) for MCS and 


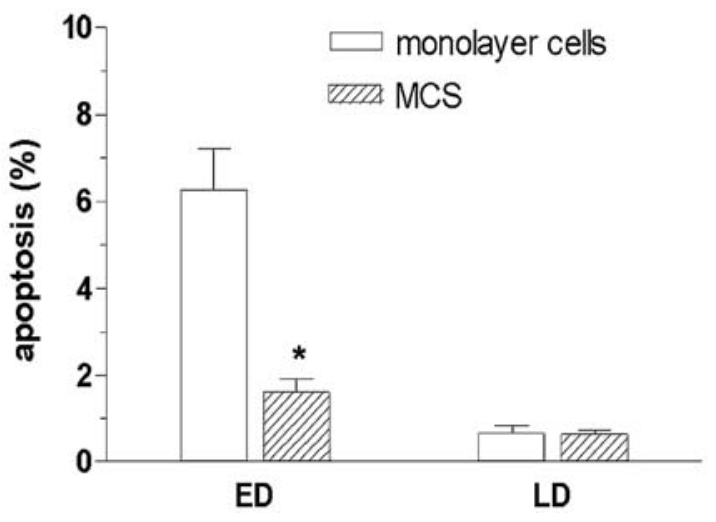

Figure 3. Cell death analyses by flow cytometry. Cell death of monolayer cultures and MCS detected at early (ED) and late (LD) time points were quantified and summarized. Data are the average of five experiments $\left({ }^{*} \mathrm{p}<0.05\right)$.

monolayer cells were $1.62 \pm 0.50 \%$ and $6.29 \pm 1.61 \%$, respectively $(\mathrm{p}<0.05)$. Late stage cell death (LD) was not significantly different between the two types of cell cultures (Fig. 3).

The drug sensitivity of MCS. The sensitivity of the cells cultured in monolayers and MCS to CDDP, 5-FU, and ADM was investigated by MTT assay. Cell inhibition ratios in monolayer cultures exposured to various concentrations of drugs were higher than those in MCS $(\mathrm{p}<0.05)$ (Fig. 4A), indicating the HepG2 cells in MCS became resistant to CDDP, 5-FU, and ADM. When drug concentrations were increased, cell inhibition ratios also increased.

On the basis of MTT assay results, an Annexin V/PI staining method was used to evaluate the apoptosis rate at 3
Table I. Immunohistochemical pictorial analysis of protein expression.

\begin{tabular}{llll}
\hline & \multicolumn{2}{c}{ Mean grey value } & \\
\cline { 2 - 3 } & Monolayer cells & \multicolumn{1}{c}{ MCS } & P \\
\hline E-cadherin & $232.28 \pm 7.80$ & $199.93 \pm 16.58$ & $<0.01$ \\
CD44v6 & $256.82 \pm 13.87$ & $207.45 \pm 8.79$ & $<0.01$ \\
VEGF & $209.81 \pm 7.11$ & $194.71 \pm 13.23$ & $<0.01$ \\
KDR & $174.40 \pm 19.12$ & $140.98 \pm 19.92$ & $<0.01$ \\
Endostatin & $162.42 \pm 6.88$ & $148.48 \pm 11.06$ & $<0.01$ \\
Cyt-C & $184.97 \pm 16.09$ & $211.12 \pm 21.02$ & $<0.05$ \\
Bax & $172.56 \pm 17.80$ & $171.76 \pm 14.69$ & $>0.05$ \\
\hline
\end{tabular}

different concentrations of each drug. As expected, apoptosis rates were much lower in MCS than those in monolayer cells after exposure to 5-FU and ADM for $48 \mathrm{~h}(\mathrm{p}<0.05)$, but were not significantly different after exposure to CDDP for the same amount of time ( $>>0.05)$ (Fig. 4B).

The protein expression profile of MCS. IHC analysis demonstrated that the cell adhesion molecules E-cadherin and CD44v6 were upregulated in MCS compared to monolayer cells (Table I). E-cadherin protein was detected predominantly in the cellular membrane and in the cytoplasm (Fig. 5A). The positive signals of CD44v6 expression were observed mainly in the cellular membrane (Fig. 5B). Further analyses in this study showed that Cyt-C levels were higher in cells in MCS than in monolayer cells. The cytoplasm of MCS was rich in mitochondria as revealed by transmission electron microscopy. Thus, cells in MCS seemed to have preserved a high level of integrity. In parallel, Bax expression
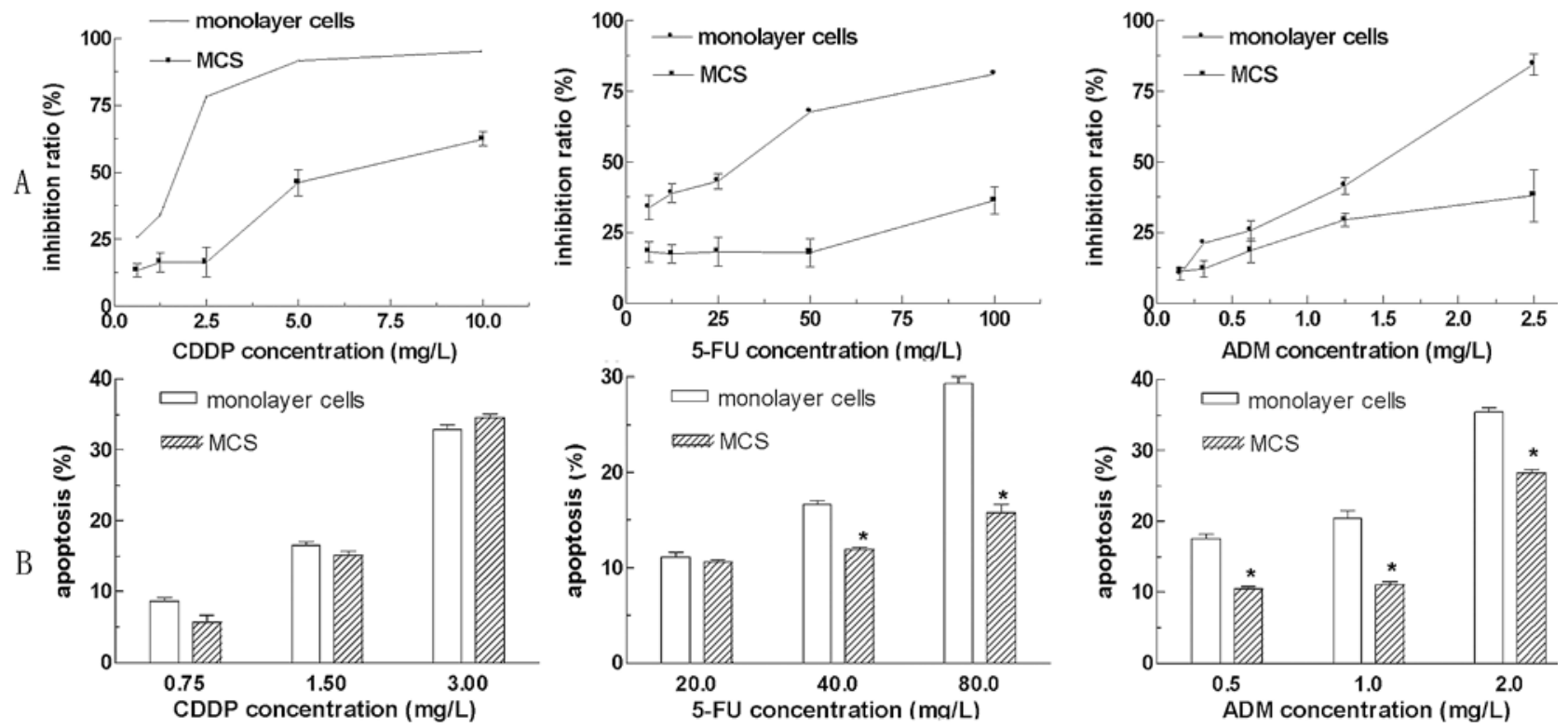

Figure 4. Drug sensitivities of HepG2 cells in a monolayer culture and MCS. (A) Cell viability measured by MTT assay. The dose-dependent effects of the indicated drugs on cell proliferation are presented as percent inhibition of cell viability. (B) Quantification of the apoptotic cell population is based Annexin $\mathrm{V}$-positive/PI-negative flow cytometry. Data represent the average of five experiments $\left({ }^{*} \mathrm{p}<0.05\right)$. 


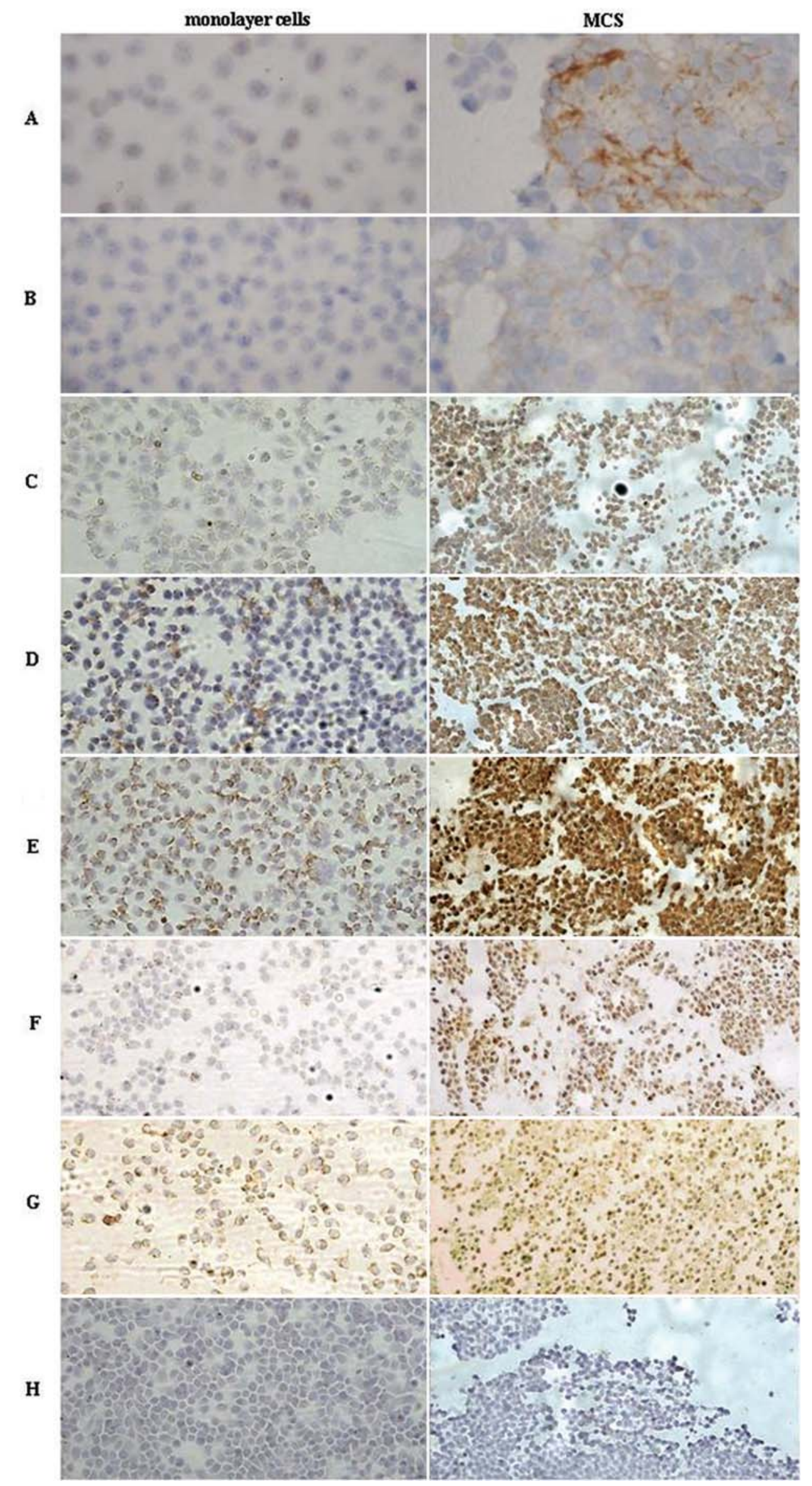

Figure 5. IHC profiling of protein expression. Representative staining results are shown for E-cadherin (A), CD44v6 (B), VEGF (C), KDR (D), endostatin $(\mathrm{E})$, cytochrome-c $(\mathrm{F})$, and Bax $(\mathrm{G})$. IHC performed with PBS in the presence of the primary antibody was used as the negative control (H). The magnifications for monolayer cultures and MCS are x200 and x400, respectively. 
was not significantly different between the two cell populations. VEGF and VEGFR-2/KDR were expressed in the cellular membrane as well as in the cytoplasm. Endostatin was expressed in the cytoplasm (Fig. 5E). In comparison with monolayer cell cultures, MCS featured elevated levels of angiogenesis-related molecules VEGF, VEGFR-2/KDR, and endostatin.

\section{Discussion}

HCC is one of the most common causes of cancer-related mortality in the world. The increasing number of patients with HCC and the highly unfavorable prognosis of the disease are two important reasons why more effort needs to be devoted to investigating more therapies able to decrease tumor progression and cancer metastasis. Systemic chemotherapy has been widely used in an attempt to prolong survival time and to provide symptomatic relief (12), but it appears to have low efficacy, possibly because it is used when metastases are already present and the tumor is large and spreading. Moreover, anticancer drug resistance occurs frequently in $\mathrm{HCC}$, and multidrug resistance (MDR) is one of the biggest limits in the use of common chemotherapies. Currently, the best understood mechanism of MDR is associated with overexpression of the protein efflux-pump known as P-glycoprotein (P-gp) (13), or of MRP1, topoisomerases, and glutathione-S transferases (14). However, this mechanism does not seem to account for all the MDR. At the center of the current study, our results suggest that earlier failures to predict drug resistance, at least in part, results from 2-D cell culture systems. Our data argue that a 3-D culture system may be superior.

MCS is a more operative model to investigate the mechanisms of drug resistance in vitro. Compared with monolayer culture, tumor cells cultured as MCS exhibit a much higher level of intrinsic or acquired MDR. Since the first studies by Sutherland et al in 1979 (15), it has been shown that tumor cells growing as MCS have many of the same behavior paterns found in solid tumors, including MDR (16). The resistance encountered in cells cultured as spheroids seems to be analogous to the natural resistance observed in patient tumors. In this study, human HepG2 cells cultured as MCS were significantly less sensitive than monolayer cells to a variety of drugs including CDDP, 5-FU, and ADM. These results are consistent with the earlier results from breast and ovarian cancer cell experiments $(17,18)$.

Our data further indicate that cells in MCS underwent significant G1 phase cell cycle arrest. Consistently, cells in more quiescent stages, such as those in MCS, appear to have more differentiated phenotypes. We showed that, in MCS of up to $160-200 \mu \mathrm{m}$ in diameter, cells were assembled with abundant tight junctions. In contrast, cells in monolayers were spread out with more membrane ruffles, but few cell junctions. These morphological differences were reported by Faute MA et al with the breast cancer cell line MCF7 (19). In light of the increased drug resistance of cells in MCS, our data suggest that cellular quiescence and differentiated phenotypes may cause drug resistance.
Although malignant cells maintain the necessary intracellular apoptotic machinery, inappropriate inhibition of apoptosis allows for continued tumor growth, metastases, and drug resistance. Our evidence that MCS formation decreased cell apoptotic sensitivity was consistent with the results of Kim et al with mesothelioma cells (20), and Denlinger et al with non-small cell lung cancer cells (21). Together these data demonstrate a direct link between drug resistance and inhibition of apoptosis in MCS.

Multicellular 3-D structures may support mechanisms of resistance to other stress stimuli. Our data, along with the published literature, demonstrate distinct features associated with 3-D cultures not found in 2-D cultures. Cells in 3-D cultures i) express more extracellular matrix proteins (22); ii) are linked by membrane protein anchorage with more cell adhesion molecules such as E-cadherin and CD44v6 (10,23-25); and iii) express more proteins involved in heat shock response or hypoxia (26-29). The overexpression of cell adhesion molecules seems to be particularly advantageous for survival, since inhibition of these molecules by specific antibodies or siRNA-mediated gene silencing disrupted the spheroid formation (10) and rendered cells more sensitive to drug-induced apoptosis (30).

MCS may favor angiogenesis. The growth and metastasis of tumors depends on angiogenesis. Pathological angiogenesis plays a pivotal role during hepatic carcinogenesis. Due to their 3-D architecture, multicellular tumor spheroids may also manifest the biochemical constraints found in a fast growing tumor mass that eventually develops the dependence on angiogenesis. Indeed, angiogenesis-related molecules VEGF, VEGFR-2, and endostatin were increased in the cells of MCS. These results were in agreement with the clinical finding that the mRNA levels of VEGF-165, VEGF-189, and KDR were higher in HCC than in chronic liver diseases $(31,32)$. It is important to point out that endostatin is a potent angiogenesis inhibitor. Interestingly, endostatin/C 18 overexpression seems to be positively correlated with VEGF expression and the progression of $\mathrm{HCC}(33,34)$ and other types of cancer (35). While earlier reports are also consistent with our finding, the mechanisms underlying the seemingly paradoxical expression profiles of endostatin and VEGFs remain elusive. It is likely that tumor angiogenesis may result from an imbalance between positive and negative regulators. Thus, in tumor progression, even when endostatin/C18 is upregulated, it may still not be sufficient to counteract the angiogenic stimuli. Nonetheless, further increase of endostatin through gene transfer in combination with doxorubicin showed tumor suppressive effects against HepG2 in an animal model (36).

In summary, we provide in vitro evidence that MCS of hepatoma cells mimic the heterogeneous multicellular structures and the more differentiated/quiescent cellular behavior that are common characteristics of solid tumors in vivo. More importantly, this MCS structure restored the resistance of tumor cells to drug-induced apoptosis. These findings call for a greater adoption of 3-D culture systems for cancer drug screening and for better mechanistic studies of MDR. 


\section{Acknowledgements}

This work was supported by the Science and Technology Investigation and Development Project of Shaanxi Province, P.R. China (to C-L Li).

\section{References}

1. Koller FL, Geevarghese SK and Gorden DL: Liver transplantation for hepatocellular carcinoma: current role and future opportunities. Curr Pharm Des 13: 3265-3273, 2007.

2. Fong Y, Sun RL, Jarnagin W and Blumgart LH: An analysis of 412 cases of hepatocellular carcinoma at a Western center. Ann Surg 229: 790-800, 1999.

3. Okuda K: Hepatocellular carcinoma. J Hepatol 32: 225-237, 2000.

4. Rougier P, Mitry E, Barbare JC and Taieb J: Hepatocellular carcinoma (HCC): an update. Semin Oncol 34: S12-S20, 2007.

5. Llovet JM, Burroughs A and Bruix J: Hepatocellular carcinoma. Lancet 362: 1907-1917, 2003

6. Llovet JM, Fuster J and Bruix J: The Barcelona approach: diagnosis, staging and treatment of hepatocellular carcinoma Liver Transpl 10: S115-S120, 2004.

7. Bokhari M, Carnachan RJ, Cameron NR and Przyborski SA: Novel cell culture device enabling three-dimensional cell growth and improved cell function. Biochem Biophys Res Commun 354: 1095-1100, 2007.

8. Desoize B and Jardilier J: Multicellular resistance: a paradigm for clinical resistance? Crit Rev Oncol Hematol 36: 193-207, 2000.

9. Hynes R: Integrins: bidirectional, allosteric signaling machines. Cell 110: 673-687, 2002.

10. Green SK, Francia G, Isidoro C and Kerbel RS: Antiadhesive antibodies targeting E-cadherin sensitize multicellular tumor spheroids to chemotherapy in vitro. Mol Cancer Ther 3:149-159, 2004.

11. Zhang X, Wang W, Yu W, Xie Y, Zhang X, Zhang Y and Ma X: Development of an in vitro multicellular tumor spheroid model using microencapsulation and its application in anticancer drug screening and testing. Biotechnol Prog 21: 1289-1296, 2005.

12. Leung TW and Johnson PJ: Systemic therapy for hepatocellular carcinoma. Semin Oncol 28: 514-520, 2001

13. Ponce de León V and Barrera-Rodríguez R: Changes in Pglycoprotein activity are mediated by the growth of a tumour cell line as multicellular spheroids. Cancer Cell Int 5: 20, 2005.

14. Perez-Tomas R: Multidrug resistance: retrospect and prospects in anti-cancer drug treatment. Curr Med Chem 13: 1859-1876, 2006.

15. Sutherland RM, Eddy HA, Bareham B, Reich K and Vanantwerp D: Resistance to adriamycin in multicellular spheroids. Int J Radiat Oncol Biol Phys 5: 1225-1230, 1979.

16. Mueller-Klieser W: Three-dimensional cell cultures: from molecular mechanisms to clinical applications. Am J Physiol 273: C1109-C1123, 1997.

17. Orlandi P, Barbara C, Bocci G, Fioravanti A, Di Paolo A, Del Tacca $M$ and Danesi R: Idarubicin and idarubicinol effects on breast cancer multicellular spheroids. J Chemother 17: 663-667, 2005

18. Lu M, Gao R, Xiao L and Wang Z: Construction of threedimensional in vitro culture model of ovarian carcinoma and the study of its multicellular drug resistance. J Huazhong Univ Sci Technolog Med Sci 26: 741-743, 2006.

19. dit Faute MA, Laurent L, Ploton D, Poupon MF, Jardillier JC and Bobichon $\mathrm{H}$ : Distinctive alterations of invasiveness, drug resistance and cell-cell organization in 3D-cultures of MCF-7, a human breast cancer cell line, and its multidrug resistant variant. Clin Exp Metastasis 19: 161-168, 2002.
20. Kim KU, Wilson SM, Abayasiriwardana KS, et al: A novel in vitro model of human mesothelioma for studying tumor biology and apoptotic resistance. Am J Respir Cell Mol Biol 33: 541-548, 2005

21. Denlinger CE, Rundall BK and Jones DR: Modulation of antiapoptotic cell signaling pathways in non-small cell lung cancer: the role of NF-kappaB. Semin Thorac Cardiovasc Surg 16: 28-39, 2004.

22. Park KH and Song SC: Morphology of spheroidal hepatocytes within injectable, biodegradable, and thermosensitive poly(organophosphazene) hydrogel as cell delivery vehicle. J Biosci Bioeng 101: 238-242, 2006.

23. Bichat F, Mouawad R, Solis-Recendez G, Khayat D and Bastian G: Cytoskeleton alteration in MCF7R cells, a multidrug resistant human breast cancer cell line. Anticancer Res 17: 3393-3402, 1997.

24. Mougel L, Tarpin M, Albert P, et al: Three-dimensional culture and multidrug resistance: effects on immune reactivity of MCF-7 cells by monocytes. Anticancer Res 24: 935-941, 2004.

25. Kang HG, Jenabi JM, Zhang J, et al: E-cadherin cell-cell adhesion in ewing tumor cells mediates suppression of anoikis through activation of the ErbB4 tyrosine kinase. Cancer Res 67: 3094-3105, 2007.

26. Jakubowicz-Gil J, Paduch R, Gawron A and KandeferSzerszen M: The effect of heat shock, cisplatin, etoposide and quercetin on Hsp27 expression in human normal and tumour cells. Folia Histochem Cytobiol 40: 31-35, 2002.

27. Rosi A, Grande S, Luciani AM, Barone P, Mlynarik V, Viti V and Guidoni L: (1H) MRS studies of signals from mobile lipids and from lipid metabolites: comparison of the behavior in cultured tumor cells and in spheroids. NMR Biomed 17: 76-91, 2004.

28. Hazlehurst LA and Dalton WS: Mechanisms associated with cell adhesion mediated drug resistance (CAM-DR) in hematopoitec malignances. Cancer Metastasis Rev 20: 43-50, 2001.

29. Wartenberg M, Ling FC, Muschen M, et al: Regulation of the multidrug resistance transporter P-glycoprotein in multicellular tumor spheroids by hypoxia-inducible factor (HIF-1) and reactive oxygen species. FASEB J 17: 503-505, 2003.

30. Ivascu A and Kubbies M: Diversity of cell-mediated adhesions in breast cancer spheroids. Int J Oncol 31: 1403-1413, 2007.

31. Iavarone M, Lampertico P, Iannuzzi F, et al: Increased expression of vascular endothelial growth factor in small hepatocellular carcinoma. J Viral Hepat 14: 133-139, 2007.

32. Mas VR, Maluf DG, Archer KJ, Yanek KC and Fisher RA: Angiogenesis soluble factors as hepatocellular carcinoma noninvasive markers for monitoring hepatitis $\mathrm{C}$ virus cirrhotic patients awaiting liver transplantation. Transplantation 84 $1262-1271,2007$.

33. $\mathrm{Hu} \mathrm{TH}$, Huang $\mathrm{CC}, \mathrm{Wu} \mathrm{CL}$, et al: Increased endostatin/ collagen XVIII expression correlates with elevated VEGF level and poor prognosis in hepatocellular carcinoma. Mod Pathol 18: 663-672, 2005

34. Alba E, Llombart A, Ribelles N and et al: Serum endostatin and $\mathrm{bFGF}$ as predictive factors in advanced breast cancer patients treated with letrozole. Clin Transl Oncol 8: 193-199, 2006.

35. Woo IS, Kim KA, Jeon HM and et al: Pretreatment serum endostatin as a prognostic indicator in metastatic gastric carcinoma. Int J Cancer 119: 2901-2906, 2006

36. Liu F, Tan G, Li J, Dong X, Krissansen GW and Sun X: Gene transfer of endostatin enhances the efficacy of doxorubicin to suppress human hepatocellular carcinomas in mice. Cancer Sci 98: 1381-1387, 2007. 